\title{
Design And Implementation of Retrieval of Images Based on Color, Text And Shape \\ Jeyalaksshmi $S^{\mathbf{a}}$ and S. Prasanna ${ }^{\mathbf{b}}$ \\ $a_{\text {Assistant Professor, Dept of IT, Vels University, Pallavaram, Chennai }}$ \\ Email : pravija.lakshmi@gmail.com \\ Associate Professor, Dept. of Computer Application, Vels University, Pallavaram, Chennai \\ Email: prasanna@velsuniv.org
}

\section{ABSTRACT:}

Retrieving the image based on the content is one of the most significant research fields in the digital image processing area. The image retrieval is becoming an innovative and quickest way of retrieving the image. Many digital images and videos are uploaded and transitioned everyday, because of the social networking medium development. Google, Yahoo and many search engines are accessing the large collection of database based on the textual annotation. These images are annotated manually with keywords, descriptions and then the images are retrieved using text-based searching methods. Therefore, performances of these systems are not satisfactory. So, we need new techniques. These techniques will easy to handle the data and can easily access the data. The CBIR method is working on the basis of particular image feature such as color, texture and shape which are derived automatically. It uses digital processing and analysis to automatically generate descriptions directly from the media data. This paper represents how the implementation of image retrieval is done based on the content.

Keywords:

CBIR, Texture, segmentation, color, shape, Feature Extraction.

\section{Introduction:}

In the cutting edge time, the fast improvement of web, private systems and advancement of mixed media innovations, numerous computerized pictures are transferred each day. With the precarious development of the quantity of advanced pictures accessible on the web and the accessibility of picture catching gadgets, for example, computerized cameras and picture scanners, the extent of computerized items is expanding quickly, in this manner effective ordering and hunting winds up noticeably fundamental down substantial picture documents[1]. Thus, many general-reason picture recovery structures had been developed. There are 3 classes of picture recovery systems: literary substance based, content-based absolutely, and semantic based absolutely [2]. In printed contentbased structures, the photos are physically commented on through content depiction. Content explanation to all depictions physically is unrealistic because of huge naming expense and the subjectivity of human recognition. To triumph over the above perils in literary substance principally based photo recovery contraption, 'ContentBased Image Retrieval (CBIR)' moved toward becoming included.

Shading is the most overwhelming and recognizing visual trademark that is widely utilized as a part of CBIR and is invariant to picture length and introductions. As customary hue capacities used in CBIR, there are shading histogram, shade correlogram, shading shape descriptor, and 
scale shade descriptor [3]. Shading histogram is the most extreme usually utilized shading portrayal plan to symbolize the worldwide element structure of a photograph, however it doesn't have any spatial information. It is invariant to interpretation and revolution of a photograph and normalizing the histogram result in scale invariance Texture is utilized to indicate the unpleasantness or coarseness of the protest floor and depicted as an example with a couple of kind of consistency. Surface capacity has been utilized as a part of different bundles beginning from business application to medicinal imaging[3]. There are various calculations for surface assessment utilized by scientists, which incorporates Gray coevents grid, Markov arbitrary field, 'concurrent vehicle backward (SAR)' rendition, universal deterioration adaptation, Gabor sifting, wavelet decay et cetera.

Shape highlights are urgent photograph highlights, despite the fact that they have now not been comprehensively used in CBIR as shade and surface highlights. Precise extraction and outline of shape data is one of the troublesome commitments in frame picture recovery [4]. Shape capacities have appeared to be valuable in bunches of space specific pictures comprising of manmade articles. For shading depictions used in many papers, be that as it may, it's miles difficult to apply frame includes when contrasted with hue and surface as a result of the incorrectness of division. The exemplary systems of portraying shape capacities are second invariants, Fourier rebuild coefficients, territory ebbs and flows, and curve period [4].

The cell phone industry goes to exchange marvelously finished the most recent couple of years with huge advances in zones of interchanges and media. Right now, the best in class work sight and sound agreeable handheld contraptions, for example, cell phones, prepared with an advanced camera and WiFi arrange association, allow accessing excessively gigantic measure of computerized media [5]. Besides, one of these intense gadgets allows new bundles. In, a client server content materialprincipally based picture recovery system for versatile stages is propelled, which offers the usefulness of substance material-based thoroughly question and surfing from portable contraptions. The proposed system offers a versatile UI and an ordinary structure, which bolsters a tremendous scope of cell gadgets.

\section{Problem definition:}

A user creating a request like "locate snap shots of Abraham Lincoln". This kind of open-ended challenge is very hard for computer systems to perform - Lincoln might not always be facing the digicam or within the identical pose. Many CBIR systems therefore typically make use of low -degree features like texture, coloration, and form. These capabilities are both utilized in mixture with interfaces that allow easier enter of the standards or with databases which have already been educated to match features (inclusive of faces, fingerprints, or form matching). However, in standard, picture retrieval requires human remarks as a way to discover higher-level ideas.

\section{Scope of the research:}

The important for a very good system to grow suitable features and required an awesome similarity set of rules to seize the contents correctly in an picture. The functioning of the system is like at the beginning the input information preferred as 
photo format then it proceed for the training and extract feature of all images saved within the database. Similarly at parallel get the consumer question picture and do the identical function extraction manner and technique it for the similarity matching.

\section{Literature review:}

\section{S. Banuchitra, Dr.K. Kungumaraj} (2016) told about the content based video retrieval (CBIR) and what basis the video retrieval techniques are done. $\mathrm{He}$ also told about the advantages and disadvantages of CBIR and its recent techniques. Several applications of CBIR in every field of life like archeology, blood cell detection, criminal investigation, Social networking sites, image search, forensic Labs, and satellite etc. The field appears to be generating interesting and valid results, even though it has so far led to afew commercial applications [6].

2. Syed Hamad Shirazi, et. Al (2016) gave a literature survey of the Content Based Image Retrieval (CBIR) techniques based totally at the Shape, Region,Texture, Color, and also review a few $r$ equipment evolved for CBIR. Some of those strategies had been single function primarily based and a number of them had been the mixtures of those functions (Texture, Color, Shape and location). Most of the studied strategies lack accuracy and are unable to get better the semantic hole between the consumer and the CBIR system. This location of CBIR systems is a hot studies area with big potential for research and development of higher photograph retrieval strategies [7].

3. Shaziya Khan and Shamaila Khan (2016) developed a retrieval system of image based on content material houses which includes shape, texture, coloration, and so on. Commonly encoded into function vectors. One of the principle blessings of the CBIR technique is the opportunity of an automatic retrieval process rather than the conventional key-word-based method. The CBIR technology has been used in several programs which includes fingerprint identification, biodiversity records structures, digital libraries, medicinal drug and historical research amongst others. This paper targets to expand a new, green tool for CBIR based totally on above point out parameters the use of MATLAB [8].

4. Rik Das, Sudeep Thepade, Subhajit Bhattacharya, and Saurav Ghosh (2016) defined a feature extraction technique by way of a way of picture binarization for improving identity and retrieval of data the use of content based totally photo reputation. The proposed approach become tested on public datasets, specifically, Wang dataset and Oliva and Torralba (OT-Scene) dataset./ Twith 3688 pix at the entire. It has outclassed the modern-day strategies in performance measures and has proven statistical significance [9].

5. M. Yasmin, M. Sharif, I. Irum, S. Mohsin (2014) proposed an green technique for photograph search and retrieval has been proposed in this examine. For this reason photographs are decomposed in equal squares of minimum $24 \times$ sixteen size and then area detection is applied to the ones decomposed elements. Pixels class is done on the idea of area pixels and internal pixels. Features are decided on from side pixels for populating the database. Moreover, coloration variations are used to cluster same color retrieved results. Precision and 
remember charges have been used as quantification measures. It may be visible from the outcomes that proposed technique shows a excellent stability of precision and recall in minimal retrieval time, executed outcomes are produced from $66 \%$-one hundred $\%$ fee for precision and sixty eight $\%-80 \%$ for do not forget [10].

\section{Existing system:}

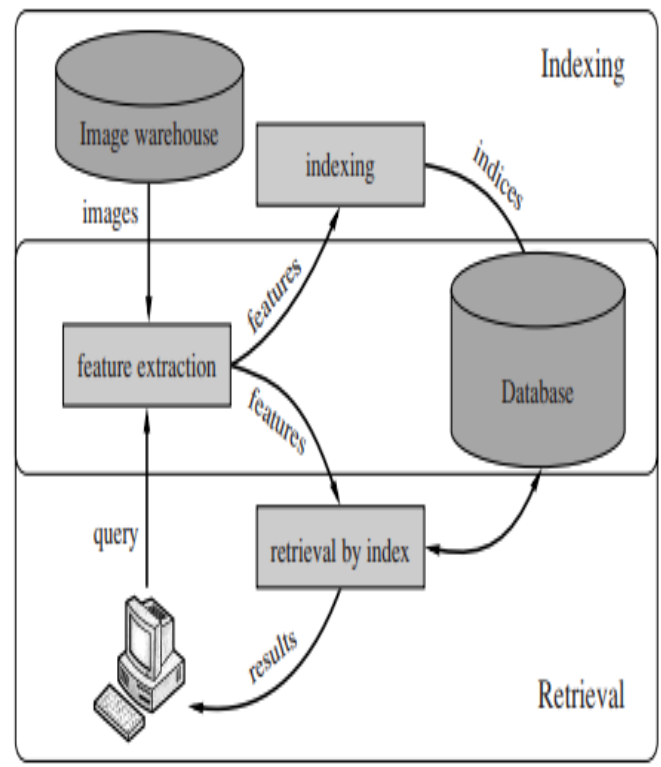

Figure 1. Existing system flow diagram

The above figure 1 clarifies the procedure stream of the current framework where the information question is given i.e., picture. At that point the component is extricated from the picture, for example, shading, shape, surface, e.t.c. Customary engineering of CBIR frameworks (Fig.1) has much in the same manner as that of traditional recovery frameworks. It likewise incorporates two fundamental modules: ordering module and recovery module. The previous handles the recovery as per the client ask. One of the CBIR frameworks key segments is that obligated for picture parameterization, i.e., an estimation of highlight vectors.. The last is responsible for developing lists and information handling, which altogether accelerate the inquiry. The contribution of this part is provided with a framework of estimations of picture pixels. The information picture is assessed, and the comparing highlight vectors of the picture are computed and sent to the ordering module. It is by these component vectors of the picture that the file is built. The segment that breaks down the highlights participates in the pursuit if the recovery depends on an example picture stacked by the client (which isn't accessible in the accumulation looked for at the initiation of the hunt procedure) [12]. It is fundamental to extricate highlights of the inquiry picture utilizing similar calculations that were utilized for extraction of the highlights while ordering a picture gathering. At that point the proposed highlight vectors of the question picture are utilized to play out the recovery. The custom way to deal with the recovery in light of the substance is to seek autonomously by various picture highlights. Each of them is spoken to by a point in the comparing highlight space. A few frameworks utilize various element spaces to represent a similar element keeping in mind the end goal to advance recovery precision [13]. For this situation, seek in each component space is additionally done autonomously, which is trailed by applying techniques for information combination to join the recovered sets (moderate yields) into one regular yield. 


\section{Proposed system:}

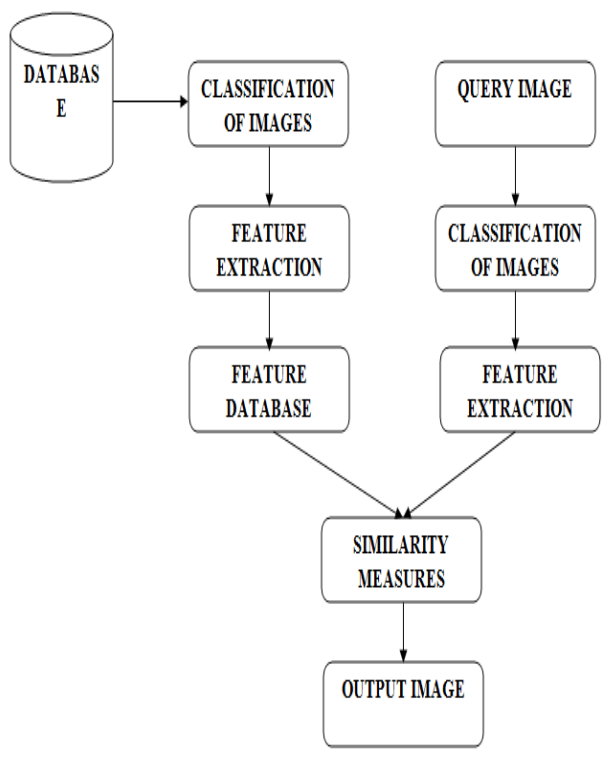

Figure 2. Proposed System Flow Chart

The above figure explains the flow chart for the proposed algorithm in which the data i.e., images are stored. The images which are kept in the database are classified according to the categories. Then the features of these images are extracted such as color, shape, texture, frame and descriptions of the particular images and stored in a database which completely consist of features.

When the input image is given. First, the image category is extracted and then the image' features are extracted. Then the similarity measures are taken between the features stored in the database and the input image' features. If any similarity found between these two, images will be retrieved.

\section{Screenshot for cbir system: \\ Database:}

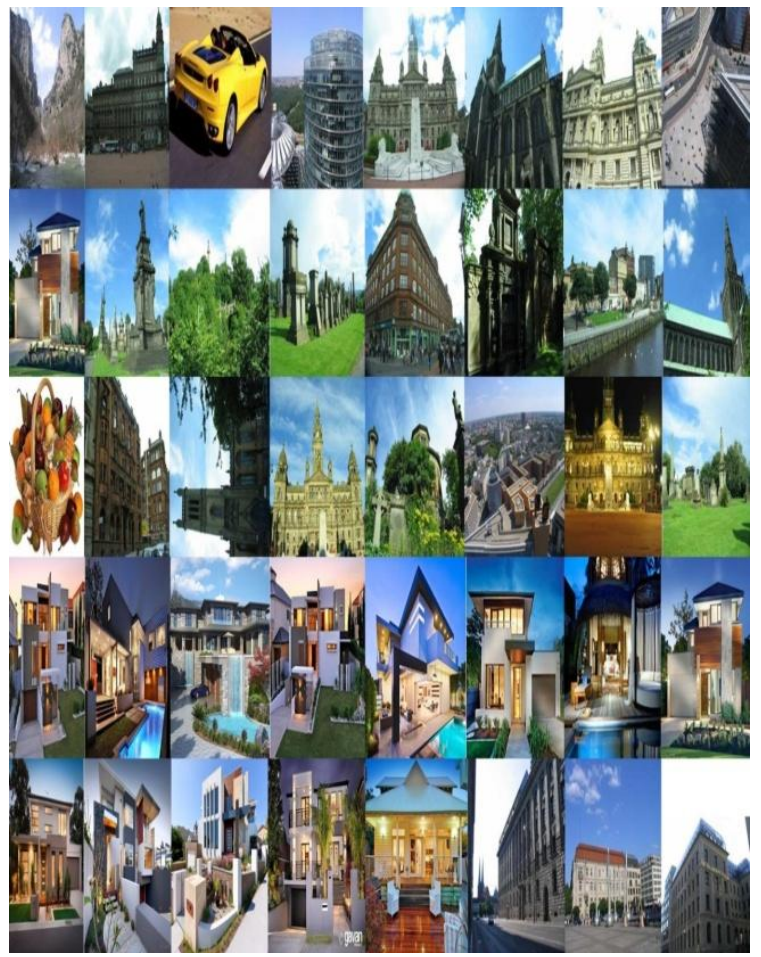

Figure. 3 Screenshot for database

The above figure 3 demonstrates the database of CBIR in which the pictures are stored.This test screenshot contains 40 pictures. For the most part, Sorting and choice is finished. In light of the info picture the CBIR works. The recovery procedure is started when a client question the framework utilizing a case picture or portray of the protest. The question picture is changed over into the interior portrayal of highlight utilizing a similar component extraction schedule that was utilized for building the element database. At long last the recovery is done by utilizing an ordering plan which plays out the productive looking in the database. As of late, client's significance input is likewise joined to additionally enhance the recovery procedure so as to deliver perceptually and semantically more important recovery comes about. 


\section{Similarity Measure:}

The decision of likeness measure influences the execution of recovery framework. The pictures are positioned by their comparability esteem and introduced as yield of CBIR framework. Numerous comparability measures have been produced in view of the quantitative assessments of the dispersion of highlights in the picture. Probably the most normally utilized comparability measures in CBIR are Euclidean separation, Minkowski-frame remove, Histogram crossing point separate, Quadratic-shape remove, Mahalanobis separation and Kullback Leibler (KL) difference remove.

\section{Input Image :}

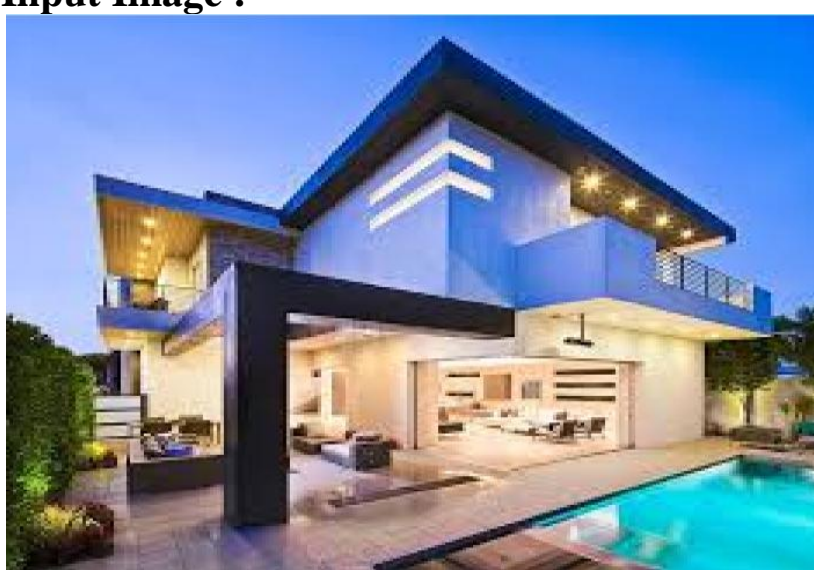

Figure 4. Input image for retrieval

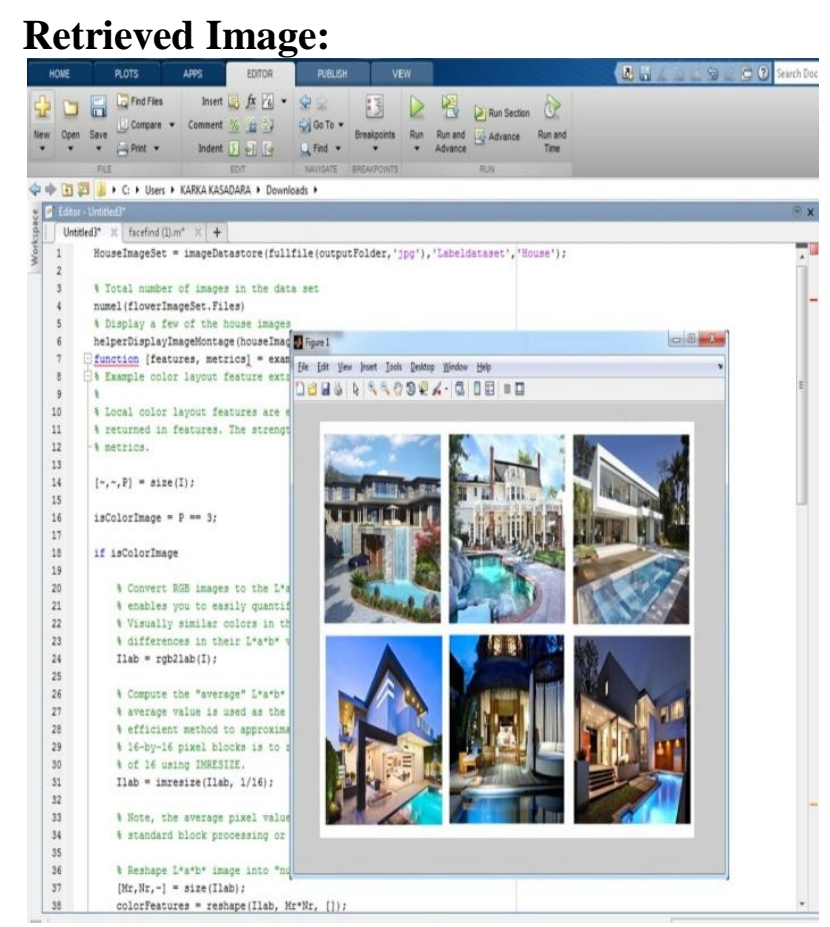

\section{Output image by CBIR.}

The above figure explains the output of content based image retrieval, In which 6 images are retrieved from the database based on color, texture, frame and shape.

\section{Conclusion:}

The most CBIR systems focus mainly on color as a primary feature for retrieval. In this research, features such as color, texture, shape, frame of an image, texture are considered. The main contribution of this research is the identification of the CBIR systems, including visual content description, similarity/distance measures, indexing scheme, user interaction. By this research, the accuracy of an image retrieval has been increased and time complexity has been reduced. 


\section{References:}

[1] M. Rehman, M. Iqbal, M. Sharif and M. Raza. Content Based Image Retrieval:Survey, World Applied Sciences Journal 19 (3) (2012): 404-412.

[2] H.+Alraqibah, M. M. Ben Ismail and O. Bchir, "Empirical Comparison of Visual Descriptors for Content based X-ray Image Retrieval", International Conference on Image and Signal Processing (ICISP'14), Cherbourg, June 2014.

[3] H. Alraqibah, O. Bchir and M. M. Ben Ismail, "X-Ray Image Retrieval System Based on Visual Feature Discrimination", Proceeding SPIE, vol. 9159, International Conference on Digital Image Processing (ICDIP), Athens, April 2014.

[4] X. Zhang, W.K. Cheung, C.H. Li, Learning latent variable models from distributed and abstracted data, Inf. Sci. Online (2013).

[5] Shereena V.B. and Julie M. David, Content Based Image Retrieval: Classification Using Neural Networks, The International Journal of Multimedia \& Its Applications (IJMA) 6(5)(2014), 1-14

[6] Ben Ismail M. M. and Bchir O., Content based Video Categorization using Relational Clustering with Local Scale Parameter, International Journal of Computer Science and Information Technology 8(1)(2016), 2746.

[7] Yang F., Matei B., Davis L. S., Reranking by Multi-feature Fusion with Diusion for Image Retrieval, Conf on Applications of Computer Vision (WACV), 2015.
[8] Hassekar P.P.; Sawant R.R., , "Experimental analysis of perceptual based texture features for image retrieval," in International Conference on Communication, Information \& Computing Technology (ICCICT), 2015.

[9] Walia E., Pal A., Fusion framework for effective color image retrieval, J. Vis. Commun. Image 25, 1335-1348, 2014.

[10] Dong J., Li X., Liao S., Xu J., Xu D., Du X., Image Retrieval by CrossMedia Relevance Fusion, Proc. of ACM MM, 2015. [88] Yang F., Matei B., Davis L. S., Re-ranking by Multi-feature Fusion with Diusion for Image Retrieval, Conf on Applications of Computer Vision (WACV), 2015.

[11] Guo J. M.; Prasetyo H.; Chen J. H., Content-Based Image Retrieval Using Error Diusion Block Truncation Coding Features, IEEE Transactions on Circuits and Systems for Video Technology, 25 (3)(2015).

[12] Thepade S.p D.; Shinde Y. D., Robust CBIR using sectorisation of hybrid wavelet transforms with Cosine-Walsh, CosineKekre, CosineHartley combinations, in International Conf on Pervasive Computing (ICPC), 2015.

[13] Gupta N.; Das S.; Chakraborti S., Extracting information from a query image, for content based image retrieval," in Eighth International Conference on Advances in Pattern Recognition (ICAPR), 2015. 